\title{
A Cost-Utility Analysis of Endovascular Aneurysm Repair for Abdominal Aortic Aneurysm
}

\author{
Yutaka Takayama, MD, PhD
}

Objective: To assess medical economic adequacy of endovascular aneurysm repair (EVAR) for abdominal aortic aneurysm (AAA).

Methods: Cost-utility analysis. A total of 21 patients with AAA treated at Ibaraki Prefectural Central Hospital in 2014 were divided into non-ruptured EVAR (Group E) and open surgery (OS) (Group O), and ruptured OS (Group R) groups, and hospital costs were aggregated with a medical accounting system. Mid-level hospital costs were estimated by a diagnosis-procedure-combination analysis system. Incremental life years were extrapolated from the results of randomized controlled trials in the UK (EVAR Trial 1 and 2), a life table, and the Pancreas Cancer Registry in Japan. Quality-adjusted life years (QALY) were estimated under the assumption of a certain quality weight.

Results: Incremental cost-effectiveness ratio (ICER) of EVAR compared with the OS was calculated to be 31.0 million yen/QALY, which is economically inadequate. ICER of EVAR compared with conservative treatment was inadequate in some subgroups of extremely old patients and in patients operated for far-advanced cancer.

Conclusion: EVAR is inadequate with respect to medical economics as a substitute for OS for patients in whom both procedures are available. The indication for EVAR in patients ineligible for OS should be different from that for surgery in usual patients with AAA. (This is a translation of J Jpn Coll Angiol 2016; 56: 123-130.)

Keywords: abdominal aortic aneurysm, endovascular aneurysm repair, cost-utility analysis, incremental cost-effectiveness ratio, extremely old age

\section{Introduction}

Endovascular aortic repair (EVAR) for abdominal aortic aneurysm (AAA) has been increasingly applied in Japan

Department of Vascular Surgery, Ibaraki Prefectural Central Hospital, Kasama, Ibaraki, Japan

Received: June 7, 2017; Accepted: June 7, 2017

Corresponding author: Yutaka Takayama, MD, PhD. Department of Vascular Surgery, Ibaraki Prefectural Central Hospital, 6528 Koibuchi, Kasama, Ibaraki 390-1793, Japan

Tel: +81-296-77-1121, Fax: +81-296-77-2886

E-mail: y-takayama@chubyoin.pref.ibaraki.jp

This is a translation of J Jpn Coll Angiol 2016; 56: 123-130. since stent grafts became covered by health insurance as a special insurance-covered medical material in 2007. Currently, the number of patients undergoing EVAR exceeds that of patients undergoing open surgery (OS). Furthermore, EVAR has been increasingly indicated for extremely old patients in whom elective surgery was abandoned, those with severe heart or respiratory dysfunction, ${ }^{1,2)}$ and cancer-bearing patients ${ }^{3)}$ because of its low-level invasiveness. Initially, EVAR was expected to reduce health expenditure because of a short hospital stay and low incidence of complications, but the rate of reduction does not exceed the high device price.

On the other hand, the Japanese government decided a probative strategy (cabinet decision on June 24, 2014) based on cost-effectiveness to determine which innovative medical techniques should be covered by health insurance and to ensure not only that patients receive the benefits of medical-field innovations but also that health insurance continues. The Special Committee for Cost-Effectiveness Assessment, part of the Central Social Insurance Medical Council, was appointed to administer this trial, which ran until March 2017 and initially included seven drugs and five medical instruments as trial items.

In this article, the medical economic usefulness of an innovative medical technique for AAA, EVAR, was examined according to the indications for the procedure and extension of its indications based on cost-utility analysis (CUA).

\section{Subjects and Methods}

Patients who underwent surgery for AAA (including iliac artery aneurysm) at Ibaraki Prefectural Central Hospital (IPCH) in 2014 were divided into the nonruptured EVAR, OS, and ruptured OS groups (Groups $\mathrm{E}, \mathrm{O}$, and $\mathrm{R}$, respectively), and the age, sex, hospital stay, prognosis, total hospitalization cost based on the diagnosis-procedure combination/per-diem payment system (DPC) and fee-for-service, and specific material expenses were obtained using electronic medical records and a medical accounting system. The DPC analysis benchmark system EVE ${ }^{\mathrm{TM}}$ (Global Health Consulting Japan, Tokyo, Japan) was used to determine the mean hospital stay in 
patients who underwent EVAR (87 institutions) or OS (95 institutions) during the same period in institutions with a $\geq 500$-bed capacity that had introduced this system $(n=822$ as of 2014), and the average hospital cost was estimated. However, parameters such as specific material expenses and coefficients of medical institutions for the DPC were adopted according to those at IPCH.

On the other hand, the incremental life year value obtained by selecting EVAR was calculated as the integrated value of differences in the survival rate, assuming that the difference in that between EVAR and OS, which is initially present as a difference $(2.5 \%)$ in 30 -day mortality rate, reduces and reaches zero $\geq 4$ years after surgery. This assumption was based on the results of a randomized controlled trial (RCT), the United Kingdom EVAR trial (EVAR-1), ${ }^{4}$ in which EVAR was compared with OS. By multiplying this value by the assumed QOL value (quality weight: QW), the incremental quality-adjusted life year (QALY) value obtained by selecting EVAR was estimated, and expenses required to obtain a QALY of 1 year (incremental cost-effectiveness ratio: ICER) were calculated.

To examine the medical economic usefulness of EVAR in OS-abandoned patients, the incremental QALY ( $\triangle$ QALY) obtained by selecting EVAR was calculated with respect to the age category by using a life table and the results of an RCT, EVAR-2,5) in which EVAR was compared with conservative treatment in patients in whom OS was considered to be physically impossible, and the ICER was calculated. In addition, to investigate the usefulness of EVAR in AAA patients with malignant tumors, the ICER of EVAR following curative surgery for pancreas cancer was calculated with respect to the stage by using the 30-day mortality rate, median postoperative life year, and 5 -year survival rate after these operations obtained in the Pancreas Cancer Registration $\left.{ }^{6}\right)$ as well as the results of the EVAR-2.

When examining these parameters, neither outpatient care expenses before surgery nor post-discharge follow-up or additional treatment costs were included.

\section{Statistical Analysis}

To simplify the model, the following aspects were assumed or approximated: (1) The QW after rupture was zero (death). (2) The risk of rupture in each AAA diameter was equivalent to the median of the range described in guidelines $^{7}$ (50-59 mm: 9\%, 60-69 mm: 15\%, and $\geq 70 \mathrm{~mm}$ : $30 \%$ per year) and did not change until death or surgery. (3) The mortality rate of AAA/post-EVAR patients at each age was calculated by adding the aneurysm-associated mortality rate to the mortality rate in a life table, and the expected life year (life expectancy) was calculated by integrating the survival rate at each age. (4) The expected prognosis after surgery for pancreatic cancer was estimated from the median when it was $\geq 5$ years and from the median and 5-year survival rate when the median was $<5$ years, considering the small number of patients surviving for a long period.

\section{Results}

The subjects consisted of 9 patients (male:female, 6:3) in Group E, 9 (7:2) in Group O, and 3 (3:0) in Group R. The mean age was $81.2 \pm 6.0$ years $($ mean $\pm S D)$ in Group E, $75.2 \pm 5.2$ years in Group $\mathrm{O}$, and $77.3 \pm 8.5$ years in Group R. All patients survived and were discharged with a mean admission period of $10.8 \pm 2.3,14.9 \pm 6.2$, and $13.7 \pm 4.2$ days, respectively. The mean total hospitalization cost based on the DPC was 2.93, 1.82, and 2.57 million yen, respectively; whereas the cost based on fee-forservice was $2.84,1.74$, and 2.27 million yen, respectively. The specific material expenses were 1.67, 0.19, and 0.17 million yen, respectively. The mean stent graft (including extension) price was 1.38 million yen in Group E, whereas the mean artificial blood vessel price was 0.15 million yen in Groups $\mathrm{O}$ and R (Fig. 1).

Of institutions participating in the EVE system, the mean DPC cost for EVAR and OS in institutions with a $\geq 500$-bed capacity was estimated to be 3.22 and 1.98 million yen, respectively (Table 1). Unless otherwise described, these estimated values were used as standard costs. In IPCH, the mean admission period in both the EVAR and the OS patients was shorter and the hospital cost calculated by subtracting fee-for-service from the DPC was greater than the corresponding mean values of the institutions participating in the EVE system (Fig. 2).

On the other hand, the incremental expected life year

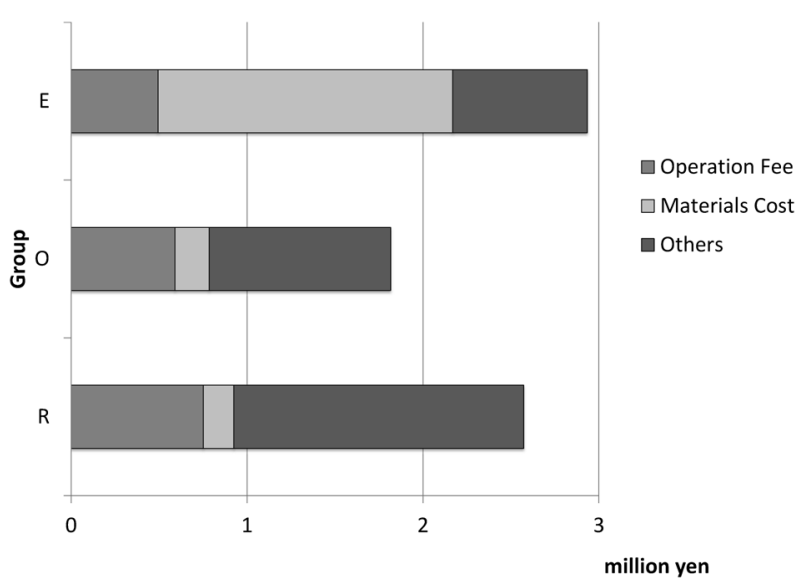

Fig. 1 Hospital costs breakdown in Group E (non-ruptured, EVAR), Group O (non-ruptured, open surgery) and Group $\mathrm{R}$ (ruptured, open surgery). The cost of materials in Group E stood out from those in the other groups and pushed up the total hospital cost. 


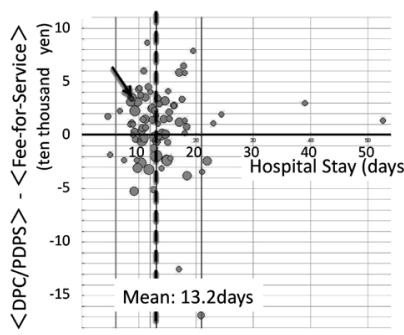

EVAR

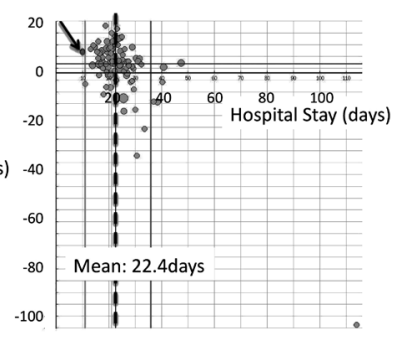

OS
Fig. 2 Hospital stay and the cost of EVAR and open surgery (OS) for AAA at the institutes participating in diagnosisprocedure-combination (DPC) analysis system EVE. The vertical axis indicates the difference between the DPC/perdiem payment system and fee-for-service-based cost (10,000 yen), and the horizontal axis indicates mean hospital stay (days). The size of a circle is proportional to the number of cases. Broken lines show mean hospital stay, and arrows show circles for Ibaraki Prefectural Central Hospital.

\section{ICER by Age}

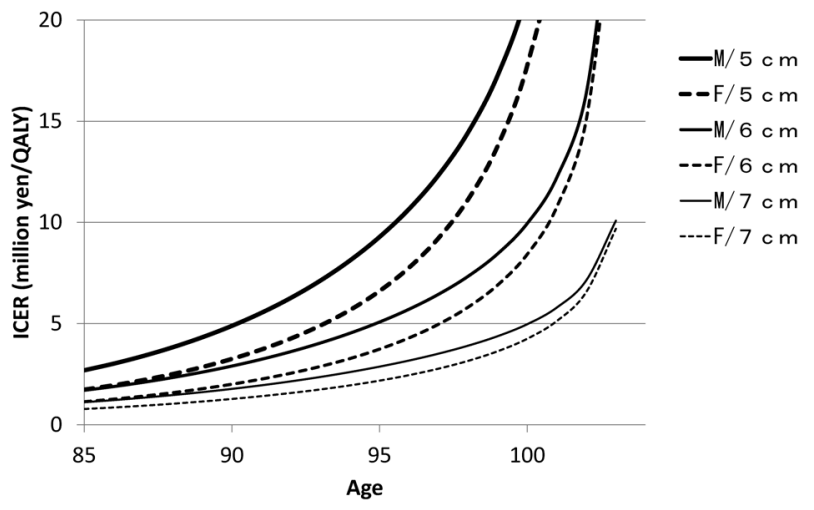

Fig. 3 Incremental cost-effectiveness ratios of EVAR by age according to sex and AAA size. Solid lines indicate males, and broken lines indicate females.

obtained by selecting EVAR was calculated as 0.050 years using the results of the EVAR-1. Assuming the mean QW during survival after surgery as $1,0.8$, and 0.6 , the ICER was calculated as $24.8,31.0$, and 41.3 million yen/QALY, respectively. Furthermore, the ICER was calculated as 22.2, 27.8, and 37.0 million yen/QALY, respectively, using the DPC results in IPCH, in which costs may be relatively low (Table 1).

The ICER in elderly patients and those with malignant tumors was calculated regarding conservative treatment as a control procedure and assuming the QW after surgery or during conservative treatment as 0.8 . When calculating the $\triangle$ QALY and ICER of EVAR for AAA of $50-59 \mathrm{~mm}$ diameter with respect to age and sex, the $\triangle$ QALY in male (female) patients aged 85, 90, and 95 years was 1.20 (1.85), 0.65 (0.99), and 0.35 (0.87) QALY, respectively.
Table 1 Hospital stay and cost of EVAR and open surgery for AAA in institutes participating in DiagnosisProcedure-Combination analysis system EVE and Ibaraki Prefectural Central Hospital, and incremental cost-effectiveness ratio calculated based on them

\begin{tabular}{|c|c|c|c|c|}
\hline & & \multirow{2}{*}{$\begin{array}{l}\text { Average institute* } \\
\text { DPC/PDPS }{ }^{6}\end{array}$} & \multicolumn{2}{|c|}{ Low-cost institute\# } \\
\hline & & & $\begin{array}{c}\text { DPC/ } \\
\text { PDPS }\end{array}$ & $\begin{array}{c}\text { Fee-for- } \\
\text { Service basis }\end{array}$ \\
\hline \multirow[t]{2}{*}{ Hospital stay } & (day) (EVAR) & 13.2 & \multicolumn{2}{|c|}{10.8} \\
\hline & (OS) & 22.4 & \multicolumn{2}{|c|}{14.9} \\
\hline \multirow[t]{2}{*}{ Hospital cost ${ }^{\&}$} & (EVAR) & 3.22 & 2.93 & 2.83 \\
\hline & (OS) & 1.98 & 1.82 & 1.74 \\
\hline$\Delta$ Cost $^{\&}$ & (EVAR-OS) & 1.24 & 1.11 & 1.09 \\
\hline \multirow[t]{3}{*}{ ICER $^{\natural}$} & $(Q W=1.0)$ & 24.8 & 22.2 & 21.8 \\
\hline & $(Q W=0.8)$ & 31.0 & 27.8 & 27.3 \\
\hline & $(Q W=0.6)$ & 41.3 & 37.0 & 36.3 \\
\hline
\end{tabular}

*Mean of the EVE Participation Institutes, \#lbaraki Prefectural Central Hospital, bDiagnosis Procedure Combination/Per-Diem Payment System, \&(million yen), ${ }^{\natural}$ incremental cost-effectiveness ratio (million yen/QALY), EVAR: endovascular aneurysm repair; OS: open surgery; QW: quality weight

Table 2 Incremental cost-effectiveness ratio of EVAR for patients operated on for pancreas cancer

\begin{tabular}{|c|c|c|c|c|c|c|}
\hline & \multirow{2}{*}{ AAA size } & \multicolumn{5}{|c|}{ Stage of pancreas cancer } \\
\hline & & I & II & III & IVa & $\mathrm{IVb}$ \\
\hline Median LY* & & 6.34 & 3.60 & 2.56 & 1.08 & 0.59 \\
\hline Expected $L Y^{*}$ & & 6.34 & 5.40 & 3.07 & 2.06 & 1.61 \\
\hline \multirow[t]{3}{*}{ ICER\# } & $5.0-5.9 \mathrm{~cm}$ & 3.59 & 4.48 & 24.00 & 127.10 & 198.43 \\
\hline & $6.0-6.9 \mathrm{~cm}$ & 2.14 & 2.43 & 8.73 & 25.68 & 47.13 \\
\hline & $\geqq 7 \mathrm{~cm}$ & 1.20 & 1.34 & 4.11 & 8.98 & 14.19 \\
\hline
\end{tabular}

*life years, \#incremental cost-effectiveness ratio (million yen/QALY)

The ICER was 2.69 (1.74), 4.89 (3.25), and 9.27 (6.61) million yen/QALY, respectively. The ICER values of EVAR for AAA of $60-69 \mathrm{~mm}$ diameter and $\geq 70 \mathrm{~mm}$ diameter with respect to age and sex are shown in Fig. 3. The ICER of EVAR for AAA of $50-59 \mathrm{~mm}$ diameter following curative surgery for pancreatic cancer was 3.59 million yen/ QALY in Stage I patients, and 127.1 million yen/QALY in Stage IVa patients. In Stage II and IVa pancreatic cancer patients with AAA of $\geq 70 \mathrm{~mm}$ diameter, it was 1.33 and 8.98 million yen/QALY, respectively (Table 2 ).

\section{Discussion}

With the rapid aging of society and progress in medical techniques, health expenditure has increased in advanced countries, including Japan. In the countries where medical care expenditure is disbursed by public resources or compulsory health insurance, medical techniques are assessed from the viewpoint of medical economics to maintain a medical system, and the results are utilized to evaluate whether each technique should be covered or to establish prices. In the United Kingdom, medical techniques are 
constantly assessed by the National Institute for Health and Clinical Excellence (NICE); medical techniques/drugs with an ICER of $£ 20,000$ to $£ 30,000 /$ QALY or higher are regarded as "not acceptable" and excluded from items to be covered by public assistance. ${ }^{8)}$ In 1982, Kaplan and Bush $^{9}$ ) calculated and compared the incremental costhealth year ratios (similar to ICER) of several medical techniques, and proposed that techniques with a value of $\$ 100,000 /$ year or higher were controversial. However, currently in the United States, many medical techniques with an ICER of \$50,000/QALY or higher are evaluated as inappropriate. ${ }^{10,11)}$ Furthermore, the WHO defined medical techniques with an ICER exceeding a value 3 -fold the gross domestic product (GDP) per person as "not cost-effective" in the CHOICE program. ${ }^{12)}$ When applying this to Japan, the threshold is 11.8 million yen/QALY. Shiroiwa et al. ${ }^{13)}$ reported that willingness-to-pay was 5-6 million yen/QALY on a population-based survey in Japan. These standards (thresholds) depend on the nationality, locality, social system, and economic state of the country, and should be decided between those receiving the benefits of medical practice (nations) and organizations responsible for the payment of health expenditure (insurers). However, regarding the medical system as a social common capital, these decisions must be based on academic findings of occupational specialists (including physicians) engaged in medical practice so that occupational regulations and ethics are not violated. ${ }^{14)}$ In Japan, the Special Committee for Cost-Effectiveness Assessment, which belongs to the Central Social Insurance Medical Council, was established in May 2012. Based on the cabinet decision made in June 2014, cost-effectiveness analysis using the ICER was introduced as a trial until March 2016.

The results of this analysis showed that the ICER of EVAR was 31 million yen/QALY in comparison with a control, the conventional treatment method OS; the value was not acceptable according to the above criteria. In other words, at the current cost, it may be inappropriate to substitute EVAR for OS in patients for whom both EVAR and OS are available from the viewpoint of medical economics. This was also consistent with the results of analysis using expenses in institutions where treatment costs were relatively restricted as references. As one of the reasons for this, the cost of OS was also low in institutions where the cost of EVAR was restricted. However, special treatment material expenses, especially stent graft expenses, are high, and each institution's efforts to minimize health expenditure may be limited. It is impossible to neutralize markedly high device prices by shortening the admission period. To justify the adequacy of EVAR in patients for whom OS can be selected from the viewpoint of medical economics, it may be necessary to markedly reduce the reimbursement price of stent grafts.
Similar to the results of this analysis, the EVAR-1, which was conducted in the United Kingdom, indicated that expenses required for initial surgery and postoperative follow-up in EVAR were much higher than those required in OS, concluding that EVAR did not have the advantage of equal cost.4) Furthermore, no cost-effectiveness was revealed in similar RCTs, such as the ACE ${ }^{15}$ ) in France and DREAM ${ }^{16)}$ in the Netherlands. However, an RCT (OVER) ${ }^{17)}$ in the United States, where initial expenses required for EVAR are lower than those required for OS, concluded that EVAR may replace OS. ${ }^{18)}$

The minimal invasiveness of EVAR has extended its indication to patients who were previously abandoned, such as extremely old patients, those with heart or respiratory failure, and those in postoperative status for abdominal cancer. To evaluate the medical economic usefulness of EVAR in these patients, conservative treatment should be selected as a control traditional method. To examine whether EVAR should be indicated for extremely old patients, the ICER of EVAR for patients with AAA of $50-59 \mathrm{~mm}$ diameter was calculated with respect to age, using conservative treatment as a control traditional method. In 90- and 95-year-old male patients, the ICER was 4.89 and 9.29 million yen/QALY, respectively. In 95-year-old female patients, it was 6.61 million yen/ QALY. These values were evaluated as "not acceptable" as a new treatment method according to the NICE criteria. The values were calculated under the assumption that there were no age-related differences in the surgical mortality rate. If the surgical mortality rate increases with age, the effectiveness may further reduce, increasing the ICER. On the other hand, among patients with AAA of $\geq 70 \mathrm{~mm}$ diameter, the ICER in 95-year-old male (female) patients was 2.88 (2.18) million yen/QALY, suggesting the medical economic usefulness of EVAR (Fig. 3).

Furthermore, the ICER of EVAR for patients with AAA of 50-59 $\mathrm{mm}$ diameter after curative surgery for Stage III pancreatic cancer was 24 million yen/QALY, and that for those with AAA of $\geq 70 \mathrm{~mm}$ diameter was 4.11 million yen/QALY. These values were also evaluated as "not acceptable" according to the NICE criteria. The results in patients after curative surgery for pancreatic cancer, as adopted in this study, may be valuable for evaluating the usefulness of EVAR in AAA patients with diseases with a relatively unfavorable prognosis (not limited to malignant tumors). According to the WHO criteria, EVAR for AAA of $50-59 \mathrm{~mm}$ diameter in patients with an expected life year of $\leq 3$ years is "not cost-effective." In addition, EVAR for AAA of $60-69 \mathrm{~mm}$ diameter in those with an expected life year of $\leq 2$ years or AAA of $\geq 70 \mathrm{~mm}$ diameter in those with an expected life year of $\leq 1.5$ years is evaluated as not cost-effective.

In the EVAR-2, an RCT in which EVAR was compared 
with conservative treatment in patients in whom OS was considered impossible on the basis of physical findings, EVAR decreased the aneurysm-related mortality rate during an 8-year follow-up period, but there was no decrease in the total mortality rate. It was concluded that no highcost-matched advantage could be confirmed. ${ }^{5}$ ) The results of this analysis suggested that AAA treatment with EVAR was medico-economically inappropriate depending on the aneurysm diameter in elderly patients or certain patients with other diseases in whom the expected life year is short. To establish medico-economically appropriate guidelines, it may be necessary to revise criteria to uniformly indicate surgery for AAA of $\geq 50 \mathrm{~mm}$ diameter.

Currently, only EVAR for high-risk patients in whom OS is difficult is covered by health insurance in Japan. This is described in the package inserts of stent grafts. High-risk patients include elderly patients, those with cardiopulmonary complications, those with a history of laparotomy, and markedly obese patients. However, the

\section{ICER by Surgical Risk}

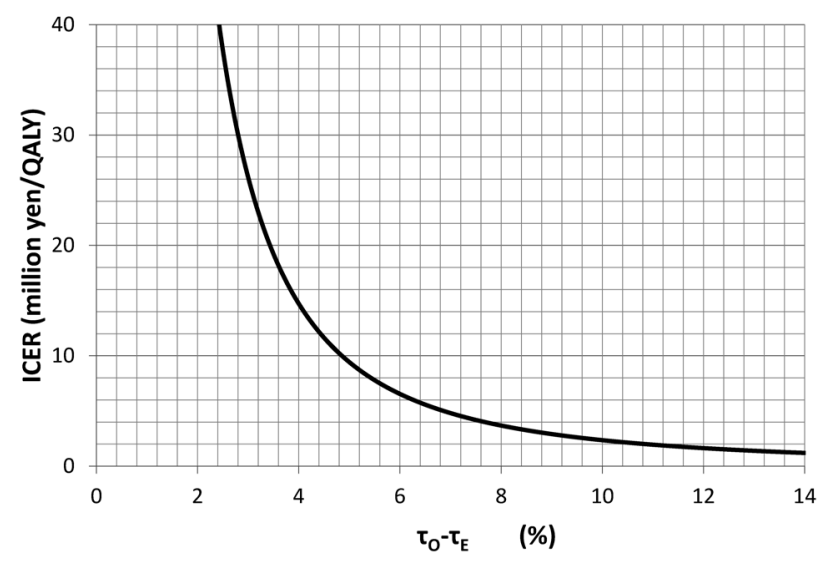

Fig. 4 Incremental cost-effectiveness ratio of EVAR by surgical risk. Horizontal axis indicates difference in operative mortality between open surgery ( $\mathrm{\tau O}$ ) and EVAR ( $\mathrm{\tau E})$. grade of risk at which EVAR is medico-economically appropriate is unclear. Figure 4 shows the ICER values with varying differences in the 30-day mortality rate between OS and EVAR. Based on the EVAR-1 results, the difference in mortality rate between EVAR and OS was assumed to reduce by $0.63 \% / y e a r$ initially, and not to exist once it became zero. According to this, when the 30 -day mortality rate of OS was $5 \%$ or $10 \%$ higher than that of EVAR, the ICER was estimated to be 9.42 or 2.36 million yen/QALY, respectively. Assuming that the 30-day mortality rate of the OS group may be 2.41-times higher than that of EVAR from the EVAR-1 results, the ICER is under the threshold (11.8 million yen/QALY) proposed in the CHOICE program (WHO) when EVAR is indicated for high-risk patients in whom the 30-day mortality rate of OS is expected to be $\geq 8.1 \%$. Using the upper limit of the NICE criteria as the threshold $(£ 30,000 / \mathrm{QALY} \approx 4.72$ million yen/QALY, mean exchange rate in April 2016), EVAR might be indicated for those with a 30-day mortality rate of OS of $\geq 12.8 \%$.

Essentially, the purpose of elective surgery for asymptomatic AAA is to prevent rupture, although it is a treatment option for the disease. Many diseases, including malignant tumors, progress in an untreated state, reducing the QW and leading to a fatal outcome. However, the presence of AAA is not considered to reduce the quality of life until rupture or death related to other diseases, excluding "anxiety," which is described below. Therefore, a treatment-related increase in the QALY is primarily related to an increase in the life year (Fig. 5). Although the QW is necessary to measure the QALY, the $\triangle$ QALY was obtained by multiplying the incremental expected life year by the assumed QW without investigation of QW in this study, considering that there is little difference in the QW of AAA patients with or without surgery and that $\triangle$ QALY obtained by EVAR mainly depends on incremental life years.
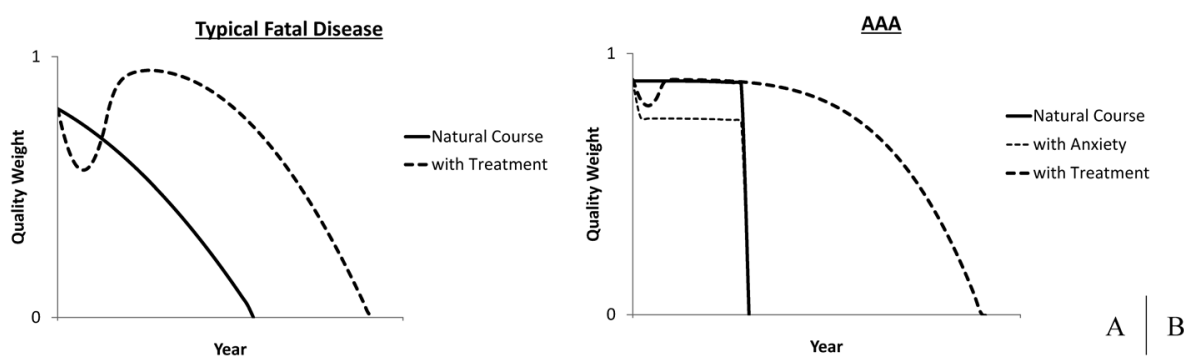

Fig. 5 Conceptual diagrams of standard processes of quality weight $(\mathrm{QW})$ in a typical fatal disease (A) and in AAA (B). A: QW gradually decreases to 0 (death) over the natural course of a typical fatal disease (solid line), whereas an effective treatment improves both QW and life span (broken line). B: QW decreases little before rupture over the natural course of AAA (solid line), whereas surgical treatment for AAA does not improve QW but only life span (bold broken line). However, anxiety about rupture might reduce $\mathrm{QW}$ over the natural course of AAA (fine broken line). 


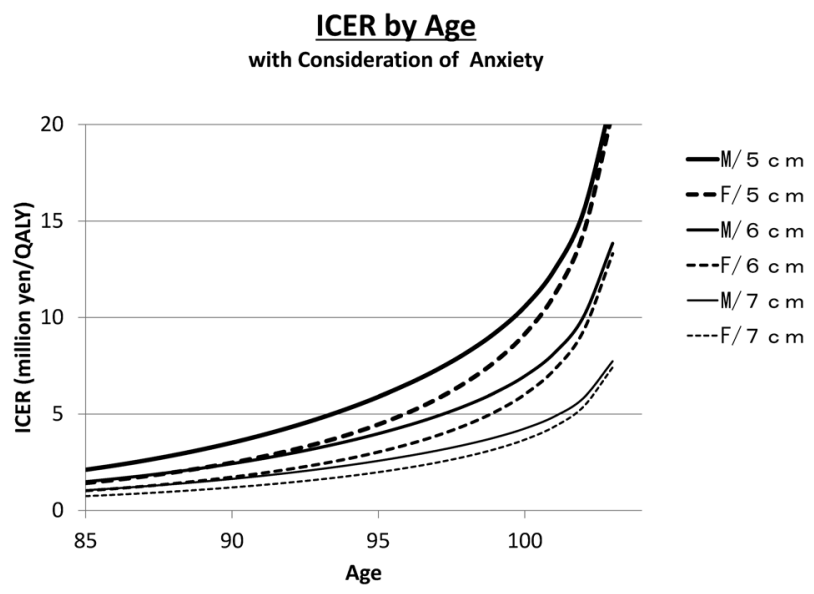

Fig. 6 Incremental cost-effectiveness ratio of EVAR by age according to sex and AAA size with the consideration of anxiety about rupture.

However, patients with untreated AAA may be anxious about rupture or subsequent death. This anxiousness may reduce the QW. Indeed, "anxiety/melancholy" is one of five dimensions in the EQ-5D instrument, a QALY measuring tool. The score of slight anxiety/melancholy is 0.072 , and that of marked anxiety/melancholy is 0.196 , which reduces the QW. ${ }^{19)}$ For example, if a patient had recognized the presence of AAA at the age of 90 years and survived with slight anxiety in an untreated state over an expected life year of 3.57 years, anxiety might have reduced the QALY by 0.26 years. Assuming that untreated AAA patients may have slight anxiety/melancholy, the ICER with respect to age was recalculated. The values in 85-, 90-, and 95-yearold male (female) patients with AAA of $50-59 \mathrm{~mm}$ diameter were 2.11 (1.43), 3.51 (2.47), and 5.89 (4.46) million yen/QALY, respectively (Fig. 6). This suggests that EVAR for 90-year-old OS-abandoned patients with AAA of $50-59 \mathrm{~mm}$ diameter is acceptable in accordance with the NICE criteria. However, these ICER values were calculated under the assumptions that there were no age-related differences in the surgical mortality rate and that anxiety/ melancholy completely disappeared after EVAR. To accurately calculate the ICER, the surgery-related mortality rate in extremely old patients must be used and QW measurement according to the degree of anxiety is necessary in untreated AAA patients or post-EVAR patients with a rupture rate of $0.5 \%-1.2 \%$ /year.

In the 1980s, during which AAA surgery for patients aged 80 years or older had not been commonly introduced, $\mathrm{Tada}^{20)}$ indicated the safety of surgery for AAA patients aged 80-89 years, and emphasized that surgical indications included smooth mental activities and dailylife restrictions related to anxiety about rupture or death. EVAR for AAA patients aged 90-99 years with an anxietyrelated reduction in the QW may be medico-economically appropriate. However, the indication regarding mental activities emphasized by Tada may also be necessary for indicating EVAR for patients of a highly advanced age.

In this study, expenses required for additional treatment in surgery-resisting patients were not included, and the postoperative QW was analyzed using assumed values. For more accurate CUA, a further study involving investigation of the short- or long-term prognosis and QW may be necessary.

\section{Conclusion}

To examine the medical economic usefulness of EVAR for AAA, cost-effectiveness analysis was performed. The ICER exceeded 20 million yen/QALY in comparison with OS as a control procedure; EVAR was not medicoeconomically acceptable. Furthermore, the ICER of EVAR for OS-abandoned patients indicated that EVAR was medico-economically inacceptable for certain elderly patients or those after surgery for cancer, depending on the aneurysm diameter. To establish medico-economically appropriate guidelines, the criteria to uniformly indicate surgery for AAA of $\geq 50 \mathrm{~mm}$ diameter should be modified.

As the above results involve many assumptions, the short- or long-term prognosis and QW should be investigated in a large number of institutions to obtain a more accurate conclusion.

\section{Disclosure Statement}

There is no conflict of interest regarding this article.

\section{Additional Note}

The protocol of this study was inspected and approved by the Clinical Research Review Committee, Ibaraki Prefectural Central Hospital, on June 24, 2015.

\section{References}

1) Bush RL, Johnson ML, Hedayati N, et al. Performance of endovascular aortic aneurysm repair in high-risk patients: results from the Veterans Affairs National Surgical Quality Improvement Program. J Vasc Surg 2007; 45: 227-33.

2) Unno N, Yamamoto N, Inuzuka $K$, et al. Outcome of endovascular repair of AAA under local anesthesia. J Jpn Soc Endovasc Intervention 2007; 8: 77-80. (in Japanese)

3) Yoshinaga K, Yoshida R, Guntani A, et al. Simultaneous total gastrectomy and endovascular repair of an abdominal aortic aneurysm: report of a case. Surg Today 2011; 41: 721-5.

4) Greenhalgh RM, Brown LC, Powell JT, et al.; United Kingdom EVAR Trial Investigators. Endovascular versus open repair of abdominal aortic aneurysm. N Engl J Med 2010; 362: 1863-71.

5) Greenhalgh RM, Brown LC, Powell JT, et al.; United 
Kingdom EVAR Trial Investigators. Endovascular repair of aortic aneurysm in patients physically ineligible for open repair. N Engl J Med 2010; 362: 1872-80.

6) Matsuno S. A summary of Pancreatic Cancer Registration for 20 years. J Jpn Pancreas Soc 2003; 18: 97-169. (in Japanese)

7) Takamoto S, Ishimaru S, Ueda Y, et al. Guidelines for diagnosis and treatment of aortic aneurysm and aortic dissection (JCS 2011). http:/www.j-circ.or.jp/guideline/pdf/JCS2011_ takamoto_h.pdf. Accessed 10 May 2016.

8) NICE article [PMG9]. Guide to the methods of technology appraisal 2013. https://www.nice.org.uk/process/pmg9/ chapter/foreword. Accessed 20 September 2016.

9) Kaplan RM, Bush JW. Health-related quality of life measurement for evaluation research and policy analysis. Health Psychol 1982; 1: 61-80.

10) Braithwaite RS, Meltzer DO, King JT Jr, et al. What does the value of modern medicine say about the $\$ 50,000$ per quality adjusted life-year decision rule? Med Care 2008; 46: 349-56.

11) Luebke T, Brunkwall J. Cost-effectiveness of endovascular versus open repair of acute complicated type B aortic dissections. J Vasc Surg 2014; 59: 1247-55.

12) World Health Organization. Cost effectiveness and strategic planning (WHO-CHOICE). http://www.who.int/choice/ costs/CER_levels/en/. Accessed 10 May 2016.

13) Shiroiwa T, Sung YK, Fukuda T, et al. International survey on willingness-to-pay (WTP) for one additional QALY gained: what is the threshold of cost effectiveness? Health Econ 2010; 19: 422-37.

14) Uzawa H. Medical Care as Social Common Capital. Tokyo: Iwanami Syoten, 2000: 167-81. (in Japanese)

15) Becquemin JP. The ACE trial: a randomized comparison of open versus endovascular repair in good risk patients with abdominal aortic aneurysm. J Vasc Surg 2009; 50: 222-4; discussion, 224.

16) De Bruin JL, Baas AF, Buth J, et al.; DREAM Study Group. Long-term outcome of open or endovascular repair of abdominal aortic aneurysm. N Engl J Med 2010; 362: 1881-9.

17) Lederle FA, Freischlag JA, Kyriakides TC, et al.; OVER Veterans Affairs Cooperative Study Group. Long-term comparison of endovascular and open repair of abdominal aortic aneurysm. N Engl J Med 2012; 367: 1988-97.

18) Epstein D, Sculpher MJ, Powell JT, et al. Long-term cost effectiveness analysis of endovascular versus open repair for abdominal aortic aneurysm based on four randomized clinical trials. Br J Surg 2014; 101: 623-31.

19) Ikeda S, Shiroiwa, Igarashi A, et al. Developing a Japanese version of the EQ-5D-5L value set. J Natl Inst Public Health 2015; 64: 47-55. (in Japanese)

20) Tada Y. Long-term follow-up results and surgical treatment of aneurysm. J Jpn Coll Angiol 1989; 29: 759-63. (in Japanese) 